\title{
Sequential Symbol Synchronizers based on Pulse Comparation at Half Rate
}

\author{
António D. Reis ${ }^{1,2}$, José F. Rocha ${ }^{1}$, Atilio S. Gameiro ${ }^{1}$, José P. Carvalho ${ }^{2}$ \\ ${ }^{1}$ Dep. de Electrónica e Telecomunicações / Instituto de Telecomunicações, Universidade de Aveiro, 3810 Aveiro, Portugal \\ ${ }^{2}$ Dep. de Fisica / U. D. Remota, Universidade da Beira Interior Covilhã, 6200 Covilhã, Portugal
}

\begin{abstract}
This work presents a synchronizer based on pulse comparation, between variable and fixed pulses. We consider four synchronizers, divided in two variants, one variant operate at the rate and the other at half rate.

Each synchronizer variant has two versions which are the manual and the automatic.

The objective is to study the synchronizers and evaluate the output jitter UIRMS (Unit Interval Root Mean Square) versus the input SNR (Signal Noise Ratio).
\end{abstract}

Keywords: Synchronism in Digital Communications

\section{INTRODUCTION}

This work studies the sequential symbol synchronizer, with a phase comparator based on a pulse comparation, between a variable pulse $\mathrm{Pv}$ and a fixed reference pulse Pf.

The synchronizer with the VCO (Voltage Controlled Oscillator) can operate at rate or at half bit rate $[1,2,3,4,5]$.

Each variant has two versions namely the manual and the automatic. The variable pulse $\mathrm{Pv}$ is produced automatically in the two versions. However, the fixed pulse is produced manually (previous human adjust) in the manual version and automatically in the automatic version[6, 7, 8, 9, 10, 11, 12].

If the clock is delayed, $\mathrm{Pv}$ is greater than $\mathrm{Pf}$, and then is applied a positive pulse $\mathrm{Pe}(\mathrm{Pv}-\mathrm{Pf})$ that advances it. On the other hand, if the clock is advanced, $\mathrm{Pv}$ is lesser than $\mathrm{Pf}$, and then is applied a negative pulse $\mathrm{Pe}(\mathrm{Pv}-\mathrm{Pf})$ that delays it.

The VCO output (clock) is a good quality version of the input synchronism information.

The clock positive transition samples the data symbols at the maximum opening eye diagram $[8,9,10,11,12]$.

Fig. 1 shows the blocks diagram of the synchronizer.

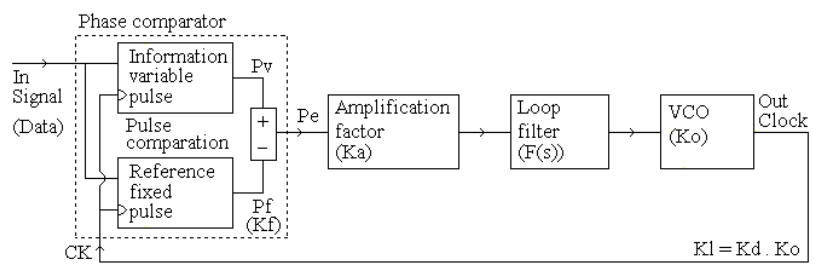

Fig.1 Synchronizer based on pulse comparation

$\mathrm{Kf}$ is the phase comparator gain, $\mathrm{F}(\mathrm{s})$ is the loop filter, Ko is the VCO gain and $\mathrm{Ka}$ is the loop amplification factor that controls the root locus and then the loop characteristics.

In priori and actual-art state was developed various synchronizers, now is necessary to know their performance.

\footnotetext{
${ }^{1 / 2} \mathrm{UA}-\mathrm{UBI}$
}

The motivation of this work is to create new synchronizers and evaluate their performance with noise. This contribution increases the know how about synchronizers.

Following, we present the variant at bit rate with their manual and automatic versions. Next, we present the variant at the half bit rate with their manual and automatic versions.

After, we present the design and tests. Then, we present the results. Finally, we present the conclusions.

\section{SYNCHRONIZERS OPERATING AT THE RATE}

The synchronizer with its VCO operates, here, at the data transmission rate.

This variant has the manual and the automatic versions, the difference in only in the phase comparator. The variable pulse $\mathrm{Pv}$ consists of first flip flop with exor and is equal in the two versions, but the fixed pulse Pf is different $[1,2]$.

\section{A. Operation at the rate and manual version}

The manual version has a phase comparator, where the fixed pulse Pf is produced by an exor with a delay $\Delta \mathrm{t}=\mathrm{T} / 2$, that needs a previous manual adjustment (Fig.2)

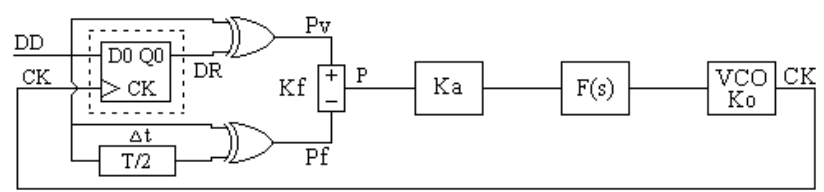

Fig.2 Synchronizer at the rate and manual (b-m)

The variable pulse $\mathrm{Pv}$ minus the fixed pulse $\mathrm{Pf}(\mathrm{Pv}-\mathrm{Pf})$ determines the error phase that controls the VCO.

Fig. 3 shows the waveforms of the synchronizer operating at the rate and manual version.

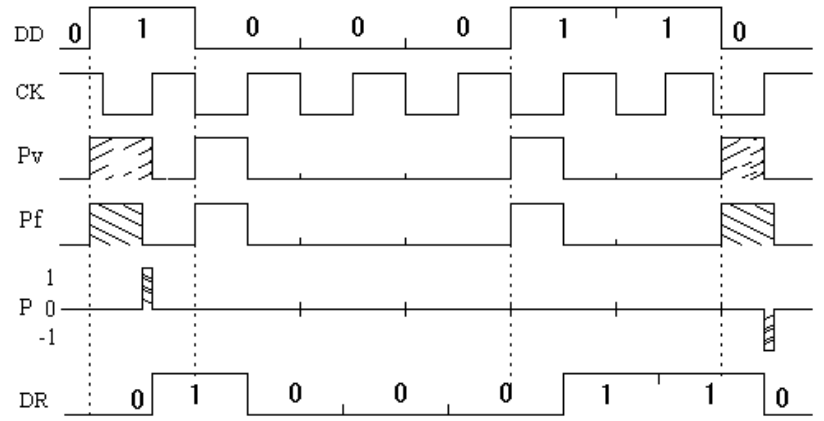

Fig.3 Waveforms of the synchronizer at the rate and manual

The error pulse Pe diminishes and disappear at the equilibrium point. 


\section{B. Operation at the rate and automatic version}

The automatic version has a phase comparator where the fixed pulse Pf is produced automatically by the second flip flop with exor, without previous adjustment (Fig.4).

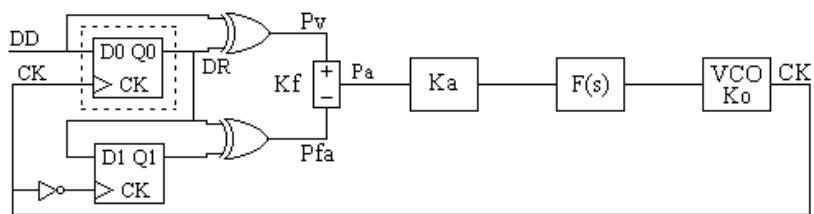

Fig.4 Synchronizer at the rate and automatic (b-a)

The variable pulse $\mathrm{Pv}$ minus the fixed pulse $\mathrm{Pf}(\mathrm{Pv}-\mathrm{Pf})$ determines the error phase that controls the VCO.

Fig. 5 shows the waveforms of the synchronizer operating at the rate and automatic version.

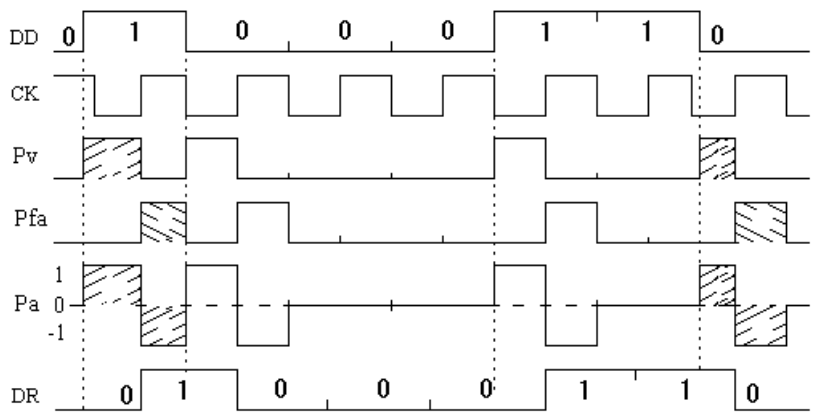

Fig.5 Waveforms of the synchronizer at the rate and automatic

The error pulse Pe don't disappear, but the variable area Pv is equal to the fixed one $\mathrm{Pf}$ at the equilibrium point.

\section{SYNCHRONIZERS OPERATING AT HALF RATE}

The synchronizer with its VCO operates, here, at half data transmission rate.

This variant has the manual and the automatic versions, but the difference is only in the phase comparator. The variable pulse $\mathrm{Pv}$, based in the two first flip flops with multiplexer, is equal in the two versions, but the fixed pulse Pf is produced from a different way $[3,4]$.

\section{A. Operation at half rate and manual version}

The manual version has a phase comparator, where the fixed pulse $\mathrm{Pf}$ is produced by an exor with a delay $\Delta \mathrm{t}=\mathrm{T} / 2$, that needs a previous manual adjustment (Fig.6).

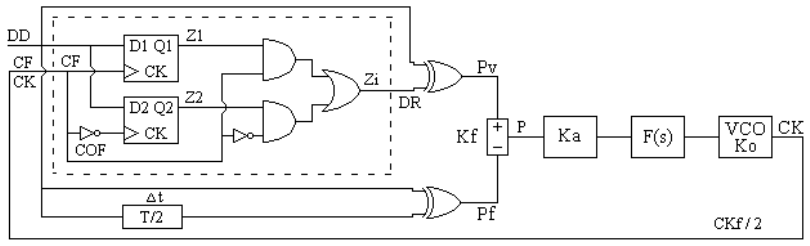

Fig.6 Synchronizer at half rate and manual (b-m/2)

The variable pulse $\mathrm{Pv}$ minus the fixed pulse Pf (Pv-Pf) determines the error phase that controls the VCO.
Fig.7 shows the waveforms of the synchronizer operating at half rate and manual version.

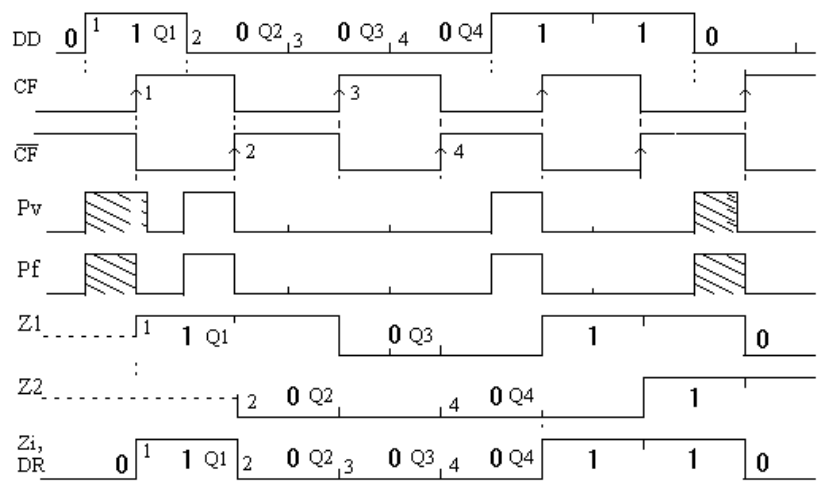

Fig.7 Waveforms of the synchronizer at half rate and manual

The error pulse Pe diminishes and disappear at the equilibrium point

\section{B. Operation at half rate and automatic version}

The automatic version has a phase comparator, where the fixed pulse Pf is produced automatically by the seconds flip flops and multiplexer with exor, without previous adjustment (Fig.8).

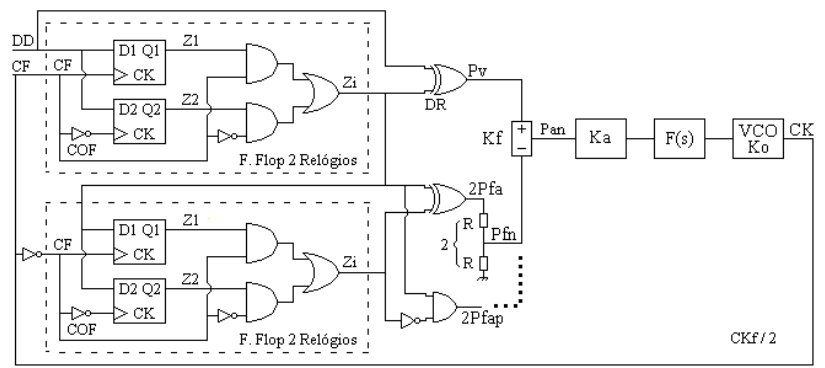

Fig.8 Synchronizer at half rate and automatic (b-a/2)

The variable pulse $\mathrm{Pv}$ minus the fixed pulse $\mathrm{Pf}(\mathrm{Pv}-\mathrm{Pf})$ determines the error phase that controls the VCO.

Fig. 9 shows the waveforms of the synchronizer at half rate and automatic version.

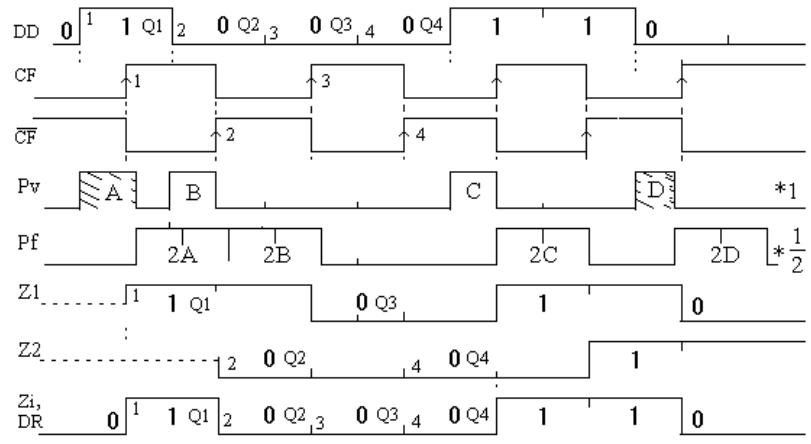

Fig.9 Waveforms of the synchronizer at half rate and automatic

The error pulse Pe don't disappear but the positive area is equal to the negative at the equilibrium point. 


\section{DESIGN, TESTS AND RESULTS}

We will present the design, the tests and the results of the referred synchronizers [5].

\section{A. Design}

To get guaranteed results, it is necessary to dimension all the synchronizers with equal conditions. Then it is necessary to design all the loops with identical linearized transfer functions.

The general loop gain is $\mathrm{Kl}=\mathrm{Kd} . \mathrm{Ko}=\mathrm{Ka} . \mathrm{Kf} . \mathrm{Ko}$ where $\mathrm{Kf}$ is the phase comparator gain, $\mathrm{Ko}$ is the $\mathrm{VCO}$ gain and $\mathrm{Ka}$ is the control amplification factor that permits the desired characteristics.

For analysis facilities, we use a normalized transmission rate $\mathrm{tx}=1$ baud, what implies also normalized values for the others dependent parameters. So, the normalized clock frequency is $\mathrm{fCK}=1 \mathrm{~Hz}$.

We choose a normalized external noise bandwidth $\mathrm{Bn}=$ $5 \mathrm{~Hz}$ and a normalized loop noise bandwidth $\mathrm{Bl}=0.02 \mathrm{~Hz}$. Later, we can disnormalize these values to the appropriated transmission rate tx.

Now, we will apply a signal with noise ratio SNR given by the signal amplitude Aef, noise spectral density No and external noise bandwidth $\mathrm{Bn}$, so the $\mathrm{SNR}=\mathrm{A}_{\mathrm{ef}}^{2} /(\mathrm{No} . \mathrm{Bn})$. But, No can be related with the noise variance $\sigma n$ and inverse sampling $\Delta \tau=1 / \mathrm{Samp}$, then $\mathrm{No}=2 \sigma \mathrm{n}^{2} . \Delta \tau$, so $\mathrm{SNR}=\mathrm{A}_{\mathrm{ef}}{ }_{\mathrm{f}} /\left(2 \sigma \mathrm{n}^{2} . \Delta \tau . \mathrm{Bn}\right)=0.5^{2} /\left(2 \sigma \mathrm{n}^{2} * 10^{-3} * 5\right)=25 / \sigma \mathrm{n}^{2}$.

After, we observe the output jitter UI as function of the input signal with noise SNR. The dimension of the loops is

\section{$-1^{\text {st }}$ order loop:}

The loop filter $\mathrm{F}(\mathrm{s})=1$ with cutoff frequency $0.5 \mathrm{~Hz}(\mathrm{Bp}=0.5$ $\mathrm{Hz}$ is 25 times bigger than $\mathrm{Bl}=0.02 \mathrm{~Hz}$ ) eliminates only the high frequency, but maintain the loop characteristics.

The transfer function is

$$
H(s)=\frac{\mathrm{G}(\mathrm{s})}{1+\mathrm{G}(\mathrm{s})}=\frac{K d K o F(s)}{s+K d K o F(s)}=\frac{K d K o}{s+K d K o}
$$

the loop noise bandwidth is

$$
\mathrm{Bl}=\frac{K d K o}{4}=K a \frac{K f K o}{4}=0.02 \mathrm{~Hz}
$$

Then, for the analog synchronizers, the loop bandwidth is $B l=0.02=(K a . K f . K o) / 4$ with $(\mathrm{Km}=1, \mathrm{~A}=1 / 2, \mathrm{~B}=1 / 2 ; \mathrm{Ko}=2 \pi)$

$($ Ka.Km.A.B.Ko $) / 4=0.02->K a=0.08 * 2 / \pi$

For the hybrid synchronizers, the loop bandwidth is $B l=0.02=(K a . K f . K o) / 4$ with $(\mathrm{Km}=1, \mathrm{~A}=1 / 2, \mathrm{~B}=0.45 ; \mathrm{Ko}=2 \pi)$

$($ Ka.Km.A.B.Ko $) / 4=0.02->K a=0.08 * 2.2 / \pi$

For the combinational synchronizers, the loop bandwidth is $B l=0.02=(K a \cdot K f . K o) / 4 \quad$ with $\quad(\mathrm{Kf}=1 / \pi ; \mathrm{Ko}=2 \pi)$

$\left(K a * 1 / \pi^{*} 2 \pi\right) / 4=0.02->K a=0.04$
For the sequential synchronizers, the loop bandwidth is $B l=0.02=(K a . K f . K o) / 4 \quad$ with $\quad(\mathrm{Kf}=1 / 2 \pi ; \mathrm{Ko}=2 \pi)$

$\left(K a * 1 / 2 \pi^{*} 2 \pi\right) / 4=0.02->K a=0.08$

The jitter depends on the RMS signal Aef, on the power spectral density No and on the loop noise bandwidth B1.

For analog PLL the jitter is

$\sigma \phi^{2}=B l . N o / A e f^{2}=B 1 \cdot 2 . \sigma n^{2} . \Delta \tau=0.02 * 10^{-3} * 2 \sigma n^{2} / 0.5^{2}=16^{*} 10^{-5} . \sigma n^{2}$

For the others PLLs the jitter formula is more complicated.

$-2^{\text {nd }}$ order loop:

The second order loop is not shown here, but the results are identical to the ones obtained above for the first order loop.

\section{B. Tests}

The following figure (Fig.10) shows the setup that was used to test the various synchronizers.

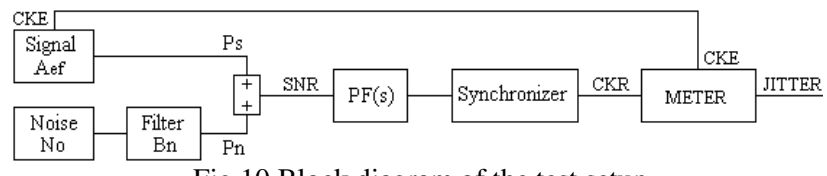

Fig.10 Block diagram of the test setup

The receiver recovered clock with jitter is compared with the emitter original clock without jitter, the difference is the jitter of the received clock.

\section{Jitter measurer (Meter)}

The jitter measurer (Meter) consists of a RS flip flop, which detects the random variable phase of the recovered clock (CKR), relatively to the fixed phase of the emitter clock (CKE). This relative random phase variation is the recovered clock jitter (Fig.11).

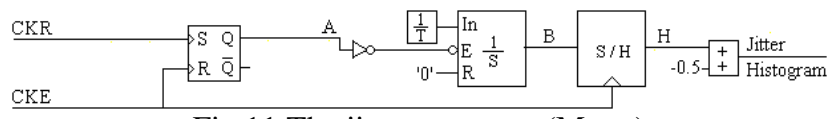

Fig.11 The jitter measurer (Meter)

The other blocks convert this random phase variation into a random amplitude variation, which is the jitter histogram. Then, the jitter histogram is sampled and processed by an appropriate program, providing the RMS jitter and the peak to peak jitter.

\section{Results}

We will present the four synchronizer results in terms of output jitter UIRMS versus input SNR.

Fig.12 shows the jitter-SNR curves of the four synchronizers using both transitions operating: at rate manual version (b$\mathrm{m})$, at rate automatic version (b-a), at half rate manual version (b-m/2) and at half rate automatic version (b-a/2). 


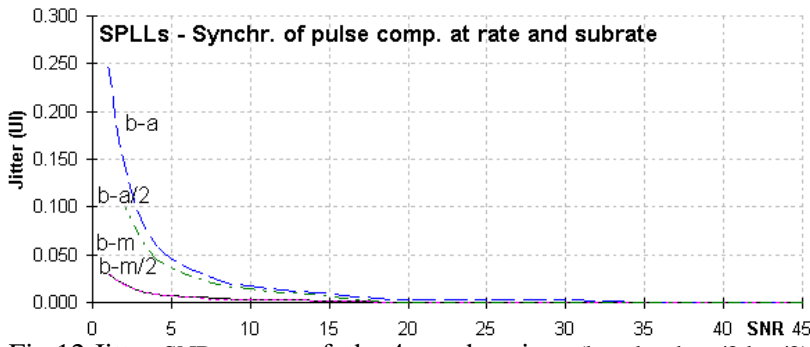

Fig.12 Jitter-SNR curves of the 4 synchronizers(b-m,b-a,b-m/2,b-a/2)

We verify that, generally, the output jitter UIRMS decreases exponentially with the input SNR increasing.

For high SNR, the four synchronizer curves tend to be similar. However, for low SNR, the manual versions (b-m, b-m/2) are similar and slightly better than the automatic versions (b-a, b-a/2).

\section{CONCLUSION AND FUTURE WORK}

We studied four synchronizers operating by both (b-) data transitions, where one variant operates at the rate (b-m, b-a) and the other one operates at half rate $(b-m / 2, b-a / 2)$. Each variant has the manual $(\mathrm{m})$ and the automatic (a) versions.

We observed that, in general, the output jitter decreases more or less exponentially with the input SNR increasing.

We verified that, for high SNR, the four synchronizers jitter tend to be similar, this is comprehensible since all the synchronizers are digital and have similar noise margin.

However, for low SNR, the manual versions (b-m, b-m/2) are significantly better than the automatic versions (b-a, b$\mathrm{a} / 2$ ), this is comprehensible since the automatic versions have more digital states than the manual versions, then the error state propagation effects caused by noise is aggravated.

In the future, we are planning to extend the present study to other types of synchronizers.

\section{ACKNOWLEDGMENTS}

The authors are grateful to the program FCT (Foundation for sCience and Technology) / POCI2010.

\section{REFERENCES}

[1] J. C. Imbeaux, "performance of the delay-line multiplier circuit for clock and carrier synchronization", IEEE Jou. on Selected Areas in Communications p.82 Jan. 1983.

[2] Werner Rosenkranz, "Phase Locked Loops with limiter phase detectors in the presence of noise", IEEE Trans. on Communications com-30 Nº10 pp.2297-2304. Oct 1982.

[3] H. H. Witte, "A Simple Clock Extraction Circuit Using a Self Sustaining Monostable Multivibrat. Output Signal”, Electronics Letters, Vol.19, Is.21, pp.897-898, Oct 1983.

[4] Charles R. Hogge, "A Self Correcting Clock Recovery Circuit”, IEEE Tran. Electron Devices p.2704 Dec 1985.

[5] A. D. Reis, J. F. Rocha, A. S. Gameiro, J. P. Carvalho "A New Technique to Measure the Jitter", Proc. III Conf. on Telecommunications pp.64-67 FFoz-PT 23-24 Apr 2001.
[6] Marvin K. Simon, William C. Lindsey, "Tracking Performance of Symbol Synchronizers for Manchester Coded Data", IEEE Transactions on Communications Vol. com-2.5 No4, pp.393-408, April 1977.

[7] J. Carruthers, D. Falconer, H. Sandler, L. Strawczynski, "Bit Synchronization in the Presence of Co-Channel Interference", Proc. Conf. on Electrical and Computer Engineering pp.4.1.1-4.1.7, Ottawa-CA 3-6 Sep. 1990.

[8] Johannes Huber, W. Liu "Data-Aided Synchronization of Coherent CPM-Receivers" IEEE Transactions on Communications Vol.40 No1, pp.178-189, Jan. 1992.

[9] Antonio D'Amico, A. D'Andrea, Reggianni, “Efficient Non-Data-Aided Carrier and Clock Recovery for Satellite DVB at Very Low SNR", IEEE Jou. on Sattelite Areas in Comm. Vol.19 Nº12 pp.2320-2330, Dec. 2001.

[10] Rostislav Dobkin, Ran Ginosar, Christos P. Sotiriou "Data Synchronization Issues in GALS SoCs", Proc. 10th International Symposium on Asynchronous Circuits and Systems, pp.CD-Ed., Crete-Greece 19-23 Apr. 2004.

[11] N. Noels, H. Steendam, M. Moeneclaey, "Effectiveness Study of Code-Aided and Non-Code-Aided ML-Based Feedback Phase Synchronizers", Proc. IEEE Int Conf. on Comm.(ICC'06) pp.2946-2951, Ist.-TK, 11-15 Jun 2006.

[12] A. D. Reis, J. F. Rocha, A. S. Gameiro, J. P. Carvalho "Effects of the Prefilter Type on Digital Symbol Synchronizers", Proc. VII Symposium on Enabling Optical Network and Sensors (SEONs 2009) pp.35-36, Lisboa (Amadora)-PT 26-26 June 2009. 